\title{
Self-heating of metallic carbon nanotube bundles in the regime of the Luttinger-liquid conductivity
}

\author{
B. A. Danilchenko, N. A. Tripachko, E. A. Voytsihovska, I. A. Obukhov, and I. I. Yaskovets
}

Institute of Physics, National Academy of Sciences of Ukraine, Prospekt Nauki, 46, Kiev 03028, Ukraine

B. Sundqvist

Department of Physics, Umea University, SE-901 87 Umea, Sweden

\begin{abstract}
:
The conductivity of bundles of carbon single-walled nanotubes with metallic conductivity (metallic nanotubes) is investigated over the wide temperature range $4.2-330 \mathrm{~K}$ and electrical fields up to $50 \mathrm{~V}$. The usage of short electrical pulses of the duration of $10 \mathrm{~ns}$ allowed to avoid an influence of a self-heating of the investigated structures on current-voltage characteristics. It is shown that the temperature dependence of conductivity is described by the power function $\mathrm{G} \propto \mathrm{T}^{\mathrm{a}}$. At helium temperatures the asymptotic dependence of current on applied voltage is close to $\mathrm{J} \propto \mathrm{V}^{1+\alpha}$ with $\alpha=0.45$. From a comparison of the obtained results of measurements with calculations, it is shown that the conductivity of nanotube bundles is well described within the theory of the Luttinger-liquid conductivity for one-dimensional conductors. The self-heating of the carbon nanotube bundles was observed in the case of measurements in the regime of dc current. A method for determination of the self-heating temperature of nanotube bundles as a function of an applied electrical field is proposed. The power dependence of the self-heating temperature on voltage $\mathrm{T} \propto \mathrm{V}^{\mathrm{p}}$ with the exponent $\mathrm{p}=$ 2.1 was observed above some threshold voltage in the temperature range 4.2-200 K. Above $200 \mathrm{~K}$ the exponent decreased down to $\mathrm{p}=1.35$.
\end{abstract}




\section{INTRODUCTION}

Carbon nanotubes (CNTs) after their discovery [1] have become the subject of intensive investigations. Electronic properties of CNTs are caused by their one-dimensionality, the character of chemical bond and the geometrical structure. So, van Hove singularities at the onset of one-dimensional energy bands confirming the one-dimensional character of conduction of CNTs, have been observed in the works (Refs. [2] and [3]). In metallic singlewalled carbon nanotubes, we are interested in, the Dirac dispersion law takes place similar to a graphene.[4] An effective carrier mass becomes zero, and, in this case, there is massless charge transport in such structures. Due to one-dimensionality, a power-law singularity of the density of states $n(q) \propto\left(q_{-}-q_{F}\right)^{a}$, where $q_{F}$ is the Fermi wave vector, appears near the Fermi energy. This state of an electron subsystem is described as a Luttinger liquid,[5-8] the conductivity of which is characterized by a non-universal Luttinger parameter a. In this case, the conductance of nanotubes $G$ depends as a power-law on the temperature $G \propto T^{\mathrm{a}}$, and the differential conductance and the current-voltage characteristics follow the universal scaling properties.[9-11] The authors in Ref. [10] have shown that the value of the parameter a depends on the type of contacts between nanotubes. The transport properties, typical for a Luttinger liquid, have been observed not only in the case of single tubes, but also in ropes of single-walled CNTs (Ref. [10]) as well as in crossing bundles of nanotubes.[11,12]

The mechanism of carrier transport in single-walled CNTs is largely determined by an interaction of electrons with lattice vibrations. This interaction shows up in different ways depending on the temperature and applied electrical fields. In weak fields, the ballistic charge transport on the distance of the order of a micrometer is observed even at room temperatures. This is caused by an interaction of carriers with acoustic vibrations in nanotubes that was demonstrated by calculations.[13]

At high voltages of the order of $0.1 \mathrm{~V}$, applied to nanotubes, the carrier transport is determined by processes of optical-phonons generation with the energy $\hbar \omega_{\text {opt }} \approx 160 \mathrm{meV}$. A mean free path of electrons decreases down to $15 \mathrm{~nm}$.[14] This point of view has been accepted in Ref. [15] to interpret experimental results. As a result of the interaction of hot carriers with optical phonons the region of negative differential conductivity (NDC) has been observed. It has been shown that the effect depends significantly on the character of heat sinking from nanotubes. The NDC has been observed in the case of limited heat sinking in freely suspended nanotubes. The NDC region has not been found in the nanotubes placed directly on a substrate.[15]

The existence of NDC opens new possibilities to use CNTs with metallic conductivity as a nonlinear element in nanoelectronic devices. Because of this, a detailed investigation of the self-heating temperature of CNTs and its dependence on an applied electrical field is an important aspect of investigations of carrier kinetics in nanotubes. The result apparently should depend on many factors such as the structural and physical state of a sample, a method of measurements and heat-sinking conditions.

In the present work, the results of investigations of conductivity of bundles of carbon singlewalled metallic nanotubes in the temperature range 4.2-330 $\mathrm{K}$ and at applied pulsed (with the pulse-length of $10 \mathrm{~ns}$ ) electrical fields up to $50 \mathrm{~V}$ are demonstrated. It is shown that the conductivity of nanotube bundles is described within the Luttinger-liquid theory for one- 
dimensional conductors up to the temperature of $195 \mathrm{~K}$. In order to investigate the selfheating of carbon nanotube bundles, the measurements were performed in a dc current regime. The comparison of pulsed measurements of current-voltage characteristics (CVC) with measurements in the regime of a dc current has evidently demonstrated the self-heating effect. A method for determination of the temperature of the nanotube bundles self-heating as a function of an applied dc electrical field has been proposed. It is shown that the temperature of the self-heating is a power function of applied voltage and dissipated power.

\section{SAMPLES AND EXPERIMENTAL TECHNIQUE}

The investigation of charge transport phenomena in structures consisting of metallic carbon nanotubes bundles were performed on samples prepared by applying uniaxial pressure. The initial powder was compressed at room temperature and under pressure of $1 \mathrm{GPa}$. According to results of the work Ref. [16], at this pressure the structure with bundles mainly oriented in the plane perpendicular to the axis of the applied pressure is formed. Nanotubes bind together by weak van der Waals forces. The initial powder used has contained $90 \%$ of single-walled carbon nanotubes. The bundle length of such nanotubes was 5-30 $\mu \mathrm{m}$. After compressing the powder the structure of the obtained sample represented a system of multiply crossing and twisted nanotubes. They form a number of contacts on intersection of nanotubes, as seen in Fig. 1 showing fragments of the structure obtained by high-resolution electron microscope.

A Raman spectroscopy of the samples made of compressed nanotubes indicated the presence of the breathing vibrational modes (RBM-mode) in the low-frequency spectrum range. The measurements were carried out at room temperature using a laser with a wave-length of 830 $\mathrm{nm}$. The spectrum fragment for the range of wave numbers $100-350 \mathrm{~cm}^{-1}$ is shown in Fig. 2 . The two intensive peaks at $\omega_{1}=234.6 \mathrm{~cm}^{-1}$ and $\omega_{2}=147 \mathrm{~cm}^{-1}$ are seen. For such values of wave numbers a diameter of nanotubes, $\mathrm{d}$, can be calculated. To do this, it is necessary to take into account the fact that the nanotubes are combined into bundles. They are kept together by van der Waals forces.

In the case of carbon nanotubes interacting in a bundle, the expression connecting the wave number $\mathrm{x}$ with the diameter $\mathrm{d}$ is written in the form Refs. [17-21],

$\omega_{\mathrm{RBM}}\left(\mathrm{cm}^{-1}\right)=\mathrm{C}_{1} / \mathrm{d}(\mathrm{nm})+\mathrm{C}_{2}$.

For the constants $\mathrm{C}_{1}$ and $\mathrm{C}_{2}$ the two data sets are used in the literature: $\mathrm{C}_{1}=224$ and $\mathrm{C}_{2}=$ $14,[19,20] \mathrm{C}^{*}{ }_{1}=239, \mathrm{C}^{*}{ }_{2}=8.5$. [18] The constant $\mathrm{C}_{2}$ accounts for the interaction between nanotubes in a bundle. Using the given set of the constants and Eq. (1), diameters of the nanotubes were calculated. It is shown that for the wave number $\omega_{1}=234.6 \mathrm{~cm}^{-1}$ there are diameters $\mathrm{d}=1.015 \mathrm{~nm}$ and $\mathrm{d}^{*}=1.057 \mathrm{~nm}$. The obtained data are close to the calculated diameter values $d=1.02 \mathrm{~nm}$ with a chirality $(\mathrm{n}=6, \mathrm{~m}=9)$ and $\mathrm{d}^{*}=1.059 \mathrm{~nm}$ with a chirality $(n=1, m=13)$. In both cases the difference $(m-n)$ is a multiple of 3 , that indicates a metallic character of conductivity of nanotubes.

The similar calculations for $\omega_{2}=147 \mathrm{~cm}^{-1}$ gave the diameter values $\mathrm{d}=1.68 \mathrm{~nm}$ and $\mathrm{d}^{*}=$ $1.726 \mathrm{~nm}$, which are close to the calculated diameters $\mathrm{d}=1.69 \mathrm{~nm}$ with a chirality $(\mathrm{n}=6$, $m=18)$ and $d^{*}=1.73 \mathrm{~nm}$ with a chirality $(n=8, m=17)$. The chirality difference, as it is in the previous case, is a multiple of 3 , hence, this type of nanotubes reveals also metallic conductivity. 
As it will be seen from results of our investigations shown below, the conductivity of structures does have a metallic character with features typical for one-dimensional metals.

Electrical measurements of such structures were performed on samples with two contacts. The samples were prepared with the shape of a dumb-bell which had a neck with a characteristic cross-section of 100_100 $\mu \mathrm{m}$ and a length of $200 \mu \mathrm{m}$. In Fig. 3, a schematic view of the sample with massive electrical contacts deposited on edges of the dumb-bell is shown. The electrical contacts were made using a conducting paste of silver powder. Such contacts were ohmic over the whole temperature range 4.2-330 K, and stable during multiple processes of cooling and heating of the sample. In the mentioned temperature range, the conductivity was studied in the constant voltage regime. The voltages applied to the sample were low enough so that the electrical resistance of the sample did not depend on the applied voltage at a given temperature.

The new methodical finding for investigations of CVC of the structures consisting of CNT bundles was the usage of short electrical pulses. Such a method of investigation was first used by the authors of the present work in Ref. [11] to study the conductivity of CNT bundles over wide ranges of temperatures and applied voltages.

The results of measurements of pulsed CVC are significantly different from the measurements in the constant voltage regime on the same sample, that will be demonstrated in what follows. A basic circuit for the CVC measurements by the pulsed method is shown in Fig. 3. A mercury relay, which discharged a coaxial cable charged up to a given voltage, was used as a generator of electrical pulses. The electrical pulses with duration of 10-30 ns were directly applied to a sample in a cryostat, and at the same time were fed to an input of one channel of a digital storage oscilloscope. The oscilloscope resolution time - $1 \mathrm{~ns}$. The second channel of the oscilloscope was registering a signal, the value of which was proportional to the current through a sample. The both signals allowed to obtain the values of the voltage on a sample and the current flowing through it.

\section{RESULTS OF MEASUREMENTS AND THEIR DISCUSSION}

The results of conductivity measurements $G$ on temperature T are shown in Fig. 4. The measurements were performed in the regime of constant voltage, the value of which was chosen not to affect the sample resistance. It is seen that the measurement results, shown in a $\log -\log$ plot, are well described by a power law $\mathrm{G} \propto \mathrm{T}^{\alpha}$ with an exponent 0.45 (the solid line in the figure) over the temperature range $25-330 \mathrm{~K}$. The power dependence of conductivity on temperature in weak electrical fields is typical for the case of the conductivity of one-dimensional systems due to the mechanism of a Tomonaga-Luttinger liquid. Such a behavior of conductivity is a consequence of the power dependence, $\mathrm{n}(\mathrm{q}) \propto\left(\mathrm{q}-\mathrm{q}_{\mathrm{F}}\right)^{\alpha}$, of the density of single-particle states near the Fermi level. Here, $q$ is the wave vector, $\mathrm{q}_{\mathrm{F}}$ is the Fermi wave vector. In the model of a Tomonaga-Luttinger liquid, the value of the nonuniversal exponent $\alpha$ is determined by strength of an electron-electron interaction within one nanotube, which, in turn, is characterized by the Luttinger interaction parameter $\beta$.[9] This parameter changes from $\beta<<1$, that corresponds to the strong elector-electron repulsion, to $\beta=1$. In this case, there is no interaction, and the condition of an existence of the classical Fermi liquid is satisfied. In a general case, the value of the exponent $\alpha$, obtained from 
transport measurements, is determined by conditions on contact interfaces as well as by an interaction between nearest nanotubes in their bundles and ropes. From investigations of photoemission spectra in similar structures consisting of CNT bundles, the value $\alpha=0.43$ was obtained at the fixed temperature of $35 \mathrm{~K}$.[22] This temperature lies in the range of values, at which, as it follows from our measurements, the existence conditions of a TomonagaLuttinger liquid are fulfilled. In the mentioned work, a contribution from external contacts of nanotubes with a metal was avoided, and the value of the quantity $\alpha$ was determined only by a contribution from internal contacts between nanotubes. Within the measurement accuracy our results coincides with the values obtained in Ref. [22]. Thus, for the chosen geometry of the sample and with taking into account the structural investigations (Fig. 1), electronic properties of the system under study are determined by multiple contacts of crossing bundles of nanotubes.

In the majority of works on the conductivity investigation of single nanotubes or their bundles, an analysis of values of $\alpha$ obtained in an experiment is based on calculations, in which an interaction between nanotubes is not taken into account. At the same time, for $\mathrm{N}$ of weakly interacting nanotubes the simple expression for the exponent $\alpha$ is proposed,[23]

$\alpha=2(1-\beta) / \mathrm{N}$

The Luttinger parameter $\beta$ is well known from calculations. In the case of single nanotubes, this parameter is equal to $0.2-0.25,[13-15]$ whereas the value $\beta=0.18$ was obtained from experimental data.[22] Using the expression (Eq. (2)), an effective number of interacting nanotubes in a bundle can be estimated. For $\mathrm{a}=0.45$ at $\beta=0.18, \mathrm{~N}=3.6$, whereas at $\beta=0.25$, $\mathrm{N}=3.3$. From these estimates it follows that electronic properties of structures consisting of bundles of crossing nanotubes are mainly caused by fragments of three or four interacting nearest nanotubes.

As it was already mentioned, in a system of carbon nanotubes the typical value for the parameter of an interaction between electrons is $\beta \approx 0.2$. This indicates a strong repulsive interaction, which can lead to break down of a metallic state and to formation of a Mott gap in the density of states in a one-dimensional conductor. In the work Ref. [24], a phase diagram for a system of interacting carbon nanotubes is considered. Depending on temperature and the parameter of exchange of carriers between nanotubes, one can expect an appearance of transitions between the states of a Mott insulator-a Luttinger liquid and phases of a FermiLuttinger liquid.

In Fig. 4, it is seen that below 25-30 K the conductivity deviates from the power dependence and falls fast with decreasing the temperature. In the range $4.2-25 \mathrm{~K}$, the conductivity has the well evident exponential dependence $\mathrm{G} \propto \exp \left[-\left(\mathrm{T}_{0} / \mathrm{T}\right)^{0.25}\right]$ with $\mathrm{T}_{0}=1950 \mathrm{~K}$ (see Fig. 5). The obtained result clearly indicates a presence of hopping conductivity with a variable hopping length that is expected for the insulating Mott phase.[24,25] Thus, we observed experimentally the expected transition from the state of a Luttinger liquid to an insulating Mott phase in the system of bundles of interacting carbon nanotubes.

The current-voltage characteristics in the pulsed regime were investigated using short electrical pulses of the duration of 10-30 ns with the pulse frequency of $100 \mathrm{~Hz}$. Such a method of investigation allowed to avoid uncontrolled selfheating of a sample in high electrical fields. The self-heating effect should be significant for the sample made up a mesh 
of nanotubes and bundles, in particular at liquid-helium temperatures. Because of CVC investigations with the usage of shot electrical pulses, we could extend significantly the range of voltages. The investigations of these characteristics were performed at several fixed temperatures within the interval 4.2-195 K. On this investigation stage, two samples with practically identical resistance at room temperature and with similar dependences of conductivity on temperature, shown in Figs. 4 and 5, were studied. The results of the CVC measurement in these samples are demonstrated in Fig. 6. It is seen that the electrical current in the structures under investigation depends significantly on the temperature.

Let us analyze the obtained results within the theory of conductivity of a Luttinger liquid. The universal relationship for dependence of current on an electrical field and temperature within the model of the Luttinger liquid has the form Refs. [26] and [27],

$\mathrm{J}=\mathrm{I}_{0} \mathrm{~T}^{\alpha+1} \operatorname{sh}\left(\mathrm{eV}_{\mathrm{s}} / 2 \mathrm{k}_{\mathrm{B}} \mathrm{T}\right)\left|\Gamma\left(1+\alpha / 2+\mathrm{i}\left(\mathrm{eV}_{\mathrm{s}} / 2 \pi \mathrm{k}_{\mathrm{B}} \mathrm{T}\right)\right)\right|^{2}$

In this expression, $\mathrm{I}_{0}$ is a constant, $\mathrm{e}$ is the electron charge, $\mathrm{k}_{\mathrm{B}}$ is the Boltzmann constant, $\Gamma(\mathrm{x})$ is a Gamma function, $V_{s}$ is the voltage drop on ends of a single nanotube. For the chosen geometry of the samples under investigation a distance between external contacts, L, on which the electrical field is concentrated, is much longer than an average distance between contacts of nanotube bundles, leff, which is determined by a structure of crossing bundles in the sample after compressing the powder (see Fig. 1). Thus, the expression for $\mathrm{V}_{\mathrm{s}}$ should be written in the form,

$\mathrm{V}_{\mathrm{s}}=\left(\mathrm{l}_{\mathrm{eff}} / \mathrm{L}\right) \mathrm{V}=\gamma \mathrm{V}$

where $\mathrm{V}$ is the drop of voltage on the sample, measured experimentally.

The expression for the current (3) has two asymptotic limits:

- a limit of low electrical fields, at which $\mathrm{eV}_{\mathrm{s}} / \mathrm{k}_{\mathrm{B}} \mathrm{T}<1$, and the expression (Eq. (3)) transforms into the power function $J / V \propto G \propto T^{\alpha}$;

- a limit of high electrical fields $\mathrm{eV}_{\mathrm{s}} / \mathrm{k}_{\mathrm{B}} \mathrm{T}>1$, which is easy realized at low enough temperatures. In this case, the dependence of current on applied voltage is described by the power function $\mathrm{J} \propto \mathrm{V}^{1+\alpha}$.

In both cases, the parameter $\alpha$ should be the same and can be obtained from independent experiments, performed at these boundary conditions. In the limit of weak electrical fields, the results of investigation of the temperature dependence of conductivity are shown in Fig. 4.

The results demonstrated in Fig. 6 show that in the limit of high electrical field the asymptotic dependence $\mathrm{J} \propto \mathrm{V}^{1+0.45}$ is observed at the temperatures 4.2 and $12 \mathrm{~K}$. An achievement of the asymptotic at much higher temperatures required higher fields, not reachable in our experimental conditions. Nonetheless, it is seen that at high temperatures all current-voltage characteristics tends to the limiting dependence.

From the expression (Eq. (3)) it follows that in the universal coordinates $\mathrm{J} / \mathrm{T}^{1+\mathrm{a}}$, depending on $\mathrm{eV}_{\mathrm{s}} / \mathrm{k}_{\mathrm{B}} \mathrm{T}$, the current-voltage characteristics measured at different temperatures should converge to one universal curve. Indeed, in Fig. 7 it is seen that in these coordinates all dependences, shown in Fig. 6, converge to a common curve. Moreover, the data for both samples coincide with each other, since they have very similar initial characteristics. The experimental results presented in the universal coordinates with the value of a free parameter 
$\gamma \approx(7-10) \cdot 10^{-4}$, are in full coincidence with the analytical expression (Eq. (3)) (in Fig. 7 it is shown by a solid line). The coincidence is observed within the wide interval of values of $\mathrm{eV}_{\mathrm{s}} / \mathrm{k}_{\mathrm{B}} \mathrm{T}$ from $10^{-2}$, that corresponds to measurements of $\mathrm{CVC}$ at $160-194 \mathrm{~K}$, up to $10^{2}$, achievable at helium temperatures. The universal scaling relationship for current, voltage and temperature follows from the model of conductivity according to the mechanism of a Luttinger liquid for a one-dimensional metal. Our experimental results shown in Figs. 4 and 7 are described by the relationship (Eq. (3)) in full measure. Hence, the conductivity of a mesh of bundles of single-walled metallic nanotubes is carried out in accordance with the mechanism of the Tomonaga-Luttinger liquid.

As it was already mentioned, all previously performed investigations of the nanotube conductivity were done using a dc current. To understand to what extent the usage of such a method is justified in the range of high electrical fields, we performed comparative measurements of CVC for different methods: pulsed and constant voltage. The measurements were done on the same sample at liquid helium temperature. The results of these comparative investigations of CVC are shown in Fig. 8. It is seen that the results of the measurements at low voltages coincide for both methods. At some voltage, in our case near $\mathrm{V}=0.1 \mathrm{~V}$, the current in the constant regime rises abruptly with respect to the pulsed measurements regime. It clearly indicates the influence of self-heating of nanotube bundles on the measured characteristics. It is obvious that depending on conditions of the experiment and the configuration of nanotubes arrangement, the self-heating can influence, in a variable degree, the results of CVC investigations.

In investigations of conductivity of carbon nanotubes, the differential conductivity $G=d J / d V$ is often studied as a function of voltage applied to nanotubes. The analytical expression of the differential conductivity in the case of a Luttinger liquid has the form,

$$
\mathrm{dJ} / \mathrm{dV}=\mathrm{AT}^{\alpha} \operatorname{ch}(\gamma \mathrm{Z})|\Gamma(1+\alpha / 2+\mathrm{i} \gamma \mathrm{Z} / \pi)|^{2} \mathrm{x}\{1-(2 / \pi) \operatorname{th}(\gamma \mathrm{Z}) \operatorname{Im}[\Psi(1+\alpha / 2+\mathrm{i} \gamma \mathrm{Z} / \pi)]\} .
$$

Here, $\mathrm{Z}=\mathrm{eV} /\left(2 \mathrm{k}_{\mathrm{B}} \mathrm{T}\right)$ is some constant, and $\Psi$ is a digamma function. The dependence of $\mathrm{G}$ on the universal energy parameter $\mathrm{eV}_{\mathrm{s}} / \mathrm{k}_{\mathrm{B}} \mathrm{T}$ allows very clearly to distinguish two intervals of an asymptotic behavior of the expression (Eq. (5)). So, at $\mathrm{eV}_{\mathrm{s}} / \mathrm{kBT} \geq 1$ the function approaches the power dependence $\mathrm{dJ} / \mathrm{dV} \propto \mathrm{V} \alpha$, where $\alpha$ is the Luttinger liquid parameter, mentioned above. In Fig. 9, the dependences of the differential conductivity based on the data in Fig. 8 are shown for the two methods of measurements. It is seen that depending on the investigation method used, there is a different exponent a in the power asymptotic function. In the case of measurements at constant voltage, we have $\alpha=1.12$; in the pulsed measurements $-\alpha=0.45$. It is just the latter value which coincides with a previously determined from the temperature dependence of conductivity (Fig. 4) and the pulsed measurements (Fig. 6). The expression (Eq. (5)) describes well the trend of the experimental dependence $\mathrm{dJ} / \mathrm{dV}$ over a wide range of variation of the energy parameter $\mathrm{eV}_{\mathrm{s}} / \mathrm{k}_{\mathrm{B}} \mathrm{T}$, which was obtained in the measurements with short pulses. The best agreement of experimental data with Eq. (5) was found at $\alpha=0.45$ and $\gamma=7 \cdot 10^{-4}$, as it is seen from Fig. 9. The observed deviation between the experimental points and the calculated dependence near $\mathrm{eV}_{\mathrm{s}} / \mathrm{k}_{\mathrm{B}} \mathrm{T} \approx 1$ can be caused by dispersion of an effective length of a nanotube $l_{\text {eff. }}$. An average value of this quantity can be obtained from the relationship $\gamma=1_{\text {eff }} / \mathrm{L}$, where $\mathrm{L}$ is the distance between external contacts, on which an applied voltage $\mathrm{V}$ is concentrated. In our case the effective sample length $\mathrm{L} \approx 200 \mu \mathrm{m}$, and $\mathrm{l}_{\text {eff }}$ is equal to about $150 \mathrm{~nm}$. The obtained length agrees well with the data of structural investigations in Fig. 1. The trend of the dependence of differential conductivity measured in the regime of constant voltage is not described by the expression (Eq. (5)) for the obtained value of $\gamma$. This 
parameter should be the same in both measurements since the investigations were performed on the same sample.

Hence, the difference in behavior of currents and differential conductivity is due to the selfheating effect of nanotube bundles when constant voltage is applied to the sample. At these conditions, the temperature of bundles becomes depending on applied voltage or electrical power. The trend of such dependence can be obtained from the data in Fig. 8 and from already established fact that the conductivity of nanotube bundles under investigation is realized in accordance with the mechanism of a Luttinger liquid. This means that in our following analyses, the analytical expression (Eq. (3)), connecting a dependence of electrical current on temperature and voltage, can be used. Using the expression (Eq. (3)), the series of currentvoltage characteristics at various temperatures was plotted. Some curves from this series are shown in Fig. 10 by solid lines. The experimental results of measurements in the regime of constant voltage are also demonstrated. In such a graph, a point of intersection of an experimental curve and calculated one at given temperature gives the voltage value at which this temperature is stabilized. For instance, the curve 4 in Fig. 10, corresponding to the temperature of $100 \mathrm{~K}$, intersects the experimental curve at the voltage of $0.7 \mathrm{~V}$. This means that at this voltage the bundle of nanotubes was self-heated up to the temperature of $100 \mathrm{~K}$. The series of such plots allowed us to determine a dependence of the temperature on constant voltage applied to a bundle of carbon nanotubes. The results are shown in Fig. 11. As seen, there exists the threshold voltage or the critical power, above which the temperature increases sharply. It should be noted that the electrical power was determined using the data of CVC measurements, shown in Fig. 10. In both cases, the temperature increase can be described by the power function $\mathrm{T} \propto \mathrm{V}^{\mathrm{p}}$ or $\mathrm{T} \propto \mathrm{W}^{\mathrm{s}}$. In the temperature range $4.2-200 \mathrm{~K}$ the values of powers $\mathrm{p}$ and $\mathrm{s}$ are equal to 2.1 and 0.75 , respectively. Above the temperature $200 \mathrm{~K}$ the exponents $\mathrm{p}$ and $\mathrm{s}$ are noticeably smaller and become equal to 1.35 and 0.5 . There are several reasons for such a change of character of the self-heating.

First, it can be expected that above $200 \mathrm{~K}$ the conductivity is not fully described by the Luttinger-liquid model. Hence, the usage of the expression (Eq. (3)) to determine the temperatures above $200 \mathrm{~K}$ can give a slightly different dependence on an electrical field. However, this deviation form the model of a Luttinger liquid seems to be small because, as seen from the data in Fig. 4, in the actual temperature range 200-330 K, the conductivity continues to change according to Eq. (3), namely: the observed dependence $\mathrm{G} \propto \mathrm{T}^{\mathrm{a}}$ can be an argument in favor of applicability of Eq. (3) up to room temperatures and somewhat above.

As the second possible cause of the abrupt change of the self-heating temperature, the change of heat sinking from bundles of nanotubes or the change of generation of acoustic phonons in the nanotubes can be considered. The thermal equilibrium in a structure of nanotube bundles is reached due to the balance of the injected electrical power and the power of heat sinking. The heat sinking is controlled by the processes of thermal conductivity and thermal exchange between nanotube bundles and an environment. Such an environment is liquid helium. At present, little is known of these thermal processes occurring in mesh structures of crossing bundles of carbon nanotubes. To resolve this question, a further detailed investigation is necessary.

Using the following consideration, one can estimate how much the character of energy losses by charge carries changes in such structures. At our experimental conditions, the maximum voltage was $2 \mathrm{~V}$ (see Fig. 8). The drop of voltage on an effective length is $\Delta \mathrm{V}=\gamma \mathrm{V}$. It was established previously that $\gamma \approx 10^{-3}$, then the value of $\Delta \mathrm{V}$ on this length is $\approx 2 \cdot 10^{-3} \mathrm{~V}$. This 
voltage is much less than a threshold of optical-phonon emission, which is $0.18 \mathrm{eV}$ for the case of an isolated carbon nanotube.[13,15] For the carriers with energies below this threshold the energy losses are due only to an acoustic-phonon emission of a spectrum of nanotube oscillations. The calculations performed in the work Ref. [13] have shown that a mean free path of charge carriers in a carbon nanotube reaches $100-160 \mathrm{~nm}$ even at room temperature. This value is close to the estimated previously effective nanotube length $1_{\text {eff }} \approx 150 \mathrm{~nm}$. Hence at low enough temperatures the carrier transport is of the ballistic character with preferential heat generation on contacts between conducting bundles of nanotubes.[28] With increasing the temperature a mean free path can become smaller than an effective length of a nanotube, and the generation of phonons shifts from contacts inward to nanotubes. In this case, the thermal exchange with an environment should also be changed. Such a redistribution of phonon generation over a nanotube length can be a cause of the observed change of the exponents $\mathrm{p}$ and $\mathrm{s}$, which characterize the self-heating of carbon nanotube bundles depending on applied constant voltage or electrical power.

It is necessary to note that in the works Refs. [15] and [29] the self-heating effect in carbon nanotubes has been studied in high electrical fields at different conditions of heatsinking. Current-voltage characteristics of nanotubes in the thermal contact with a substrate and freely suspended in a vacuum have been investigated. Experiments and calculations have been performed for carbon nanotubes with conductivity typical for a Fermi liquid. Such an approach is reasonable at high enough temperatures - of the order of room temperatures. The results of the calculation and the experiment reveal the typical for a Fermi liquid dependence of electrical current on applied voltage. In low electrical fields the current grows linearly with the field and tends to saturation at voltages above $0.1 \mathrm{~V}$. When the heat-sinking through a side surface of a nanotube decreases, the negative differential conductivity is observed.

In our case, the self-heating of nanotubes lead to a nonlinear increase of the current depending on applied constant voltage. Such a behavior is different in principle from the abovementioned literary results. The distinction is due to the fact that we have investigated the effect of self-heating in carbon nanotube bundles in the regime of a Luttinger-liquid conductivity, but not the self-heating of nanotubes with the conductivity of a Fermi liquid.

\section{CONCLUSIONS}

The results of conductivity measurements in structures of carbon nanotube bundles in the temperature range $4.2-330 \mathrm{~K}$ are presented. In the temperature range $25-330 \mathrm{~K}$ the conductivity changes according to the power law $\mathrm{G} \propto \mathrm{T}^{\alpha}$ with the exponent $\alpha=0.45$. Using short electrical pulses with a pulse duration of $10 \mathrm{~ns}$, current-voltage characteristics were investigated in the voltage range $0.5-50 \mathrm{~V}$, in which the asymptotic behavior $\mathrm{J} \propto \mathrm{V}^{1+\alpha}$ was observed at low temperatures.

All current-voltage characteristics measured in the range 4.2-200 $\mathrm{K}$ and plotted in the universal coordinates $\mathrm{J} / \mathrm{T}^{\alpha}$ as a function of $\mathrm{eV}_{\mathrm{s}} / \mathrm{k}_{\mathrm{B}} \mathrm{T}$, converge to a common curve. The scaling is observed over a wide range of values of the energy variable $\mathrm{eV}_{\mathrm{S}} / \mathrm{k}_{\mathrm{B}} \mathrm{T}$ from $10^{-2}$ to $10^{2}$ and up to the temperature of $200 \mathrm{~K}$.

The obtained result shows that the mechanism of current flowing in a mesh of carbon nanotube bundles corresponds to the model of a Luttinger liquid, in which the charge transfer is realized by charge density waves. 
In the temperature range $4.2-25 \mathrm{~K}$, the hopping conductivity with a varying hopping length was observed in these structures.

The significant difference in the behavior of CVC as well as in differential conductivities measured at $4.2 \mathrm{~K}$ using constant voltage and short electrical pulses was found. The observed difference is related to increasing the conductivity of a Luttinger liquid at the self-heating of carbon nanotube bundles by a dc current. Based on the method of determination of the bundles temperature, proposed in the work, we found the dependences of this temperature on dissipated electrical power $\mathrm{W}$ and applied voltage $\mathrm{V}$, which follow the power law: $\mathrm{T} \propto \mathrm{W}^{\mathrm{s}}$ and $\mathrm{T} \propto \mathrm{V}^{\mathrm{p}}$. The possible processes, which affect the change of the exponents $\mathrm{p}$ and $\mathrm{s}$ at the self-heating temperature above $200 \mathrm{~K}$, are discussed. 


\section{Figure captions:}

Figure 1. Different fragments of the sample structure, consisting of compressed carbon nanotube bundles, observed by electron microscope.

Figure 2. The Raman spectrum fragment in the range of wave vectors, corresponding to the breathing vibrational modes of single carbon nanotubes.

Figure 3. Main elements of electrical circuit for pulsed measurements of CVC. A schematic view of a sample with contacts.

Figure 4. Dependence of conductivity of carbon nanotube bundles $\mathrm{G}$ on temperature plotted in $\log$ - $\log$ coordinates. The solid line corresponds to the power function $\mathrm{G} \propto \mathrm{T}^{0.45}$.

Figure 5. Dependence of conductivity $\mathrm{G}$ on the inverse temperature to the power 0.25 . The straight line corresponds to the function $\mathrm{G} \propto \exp \left[-\left(\mathrm{T}_{0} / \mathrm{T}\right)\right]^{0.25}$.

Figure 6. Current-voltage characteristics in a log-log plot, measured in the pulse regime on samples at T, K: 4.2 (1), 12.5 (2), 50 (3), 78 (4), 160 (5) (a); 4.2 (1), 12 (2), 25 (3), 78 (4), 194 (5) (b). Solid lines - asymptotic functions $\mathrm{J} \propto \mathrm{V}^{1.45}$.

Figure 7. The CVC data, represented in a log-log plot, in the universal coordinates. All experimental points, corresponding to the data in Fig. 6, within the temperature range 4.2-195 $\mathrm{K}$ approaches a common curve described by the expression Eq. (3) (solid line).

Figure 8. Dependence of current on applied voltage, obtained in the regime of a dc current (curve 2), and in pulsed measurements (curve 1), performed at liquid helium temperature.

Figure 9. Differential conductivity $\mathrm{dJ} / \mathrm{dV}$ as a function of the universal energy parameter. The experimental points of the curve 1 were obtained from pulsed measurements; the curve 2 is based on experiments in the regime of a dc current. The thick solid line corresponds to the function Eq. (5). The thin solid lines - the asymptotic functions $\mathrm{dJ} / \mathrm{dV} \propto \mathrm{V}^{\alpha}$.

Figure 10. The fragment of current-voltage characteristics, measured in the regime of constant voltage ( $\square$ ). The solid lines - results of calculations according to the expression Eq. (2) at the temperatures, K: 4.2 (1), 10 (2), 20 (3), 100 (4), and 400 (5). The dotted line illustrates a correspondence of voltage and temperature of the sample self-heating.

FIG. 11. Dependence of the self-heating temperature of carbon nanotube bundles on voltage (a) and on applied electrical power (b). 


\section{References:}

[1] S. Iijima, Nature 354, 56 (1991).

[2] J. W. G. Wildöer, L. C. Venema, A. G. Rinzler, R. E. Smalley, and C. Dekker, Nature 391, 59 (1998).

[3] S. Ilani, L. A. K. Donev, M. Kindermann, and P. L. McEuen, Nature Phys. 2, 687 (2006).

[4] H. Castro Neto, F. Guinea, N. M. R. Peres, K. S. Novoselov, and A. K. Geim, Rev. Mod. Phys. 81, 109 (2009).

[5] S. Tomonaga, Prog. Theor. Phys. 5, 544 (1950).

[6] J. M. Luttinger, J. Math. Phys. 1S. Iijima, Nature 354, 56 (1991).

[7] F. D. M. Haldane, J. Phys. C 14, 2585 (1981).

[8] J. Voit, Rep. Prog. Phys. 58, 977 (1995).

[9] C. Kane, L. Balents, and M. P. A. Fisher, Phys. Rev. Lett. 79, 5086 (1997).

[10] M. Bockrath, D. H. Cobden, J. Lu, A. G. Rinzler, R. E. Smalley, T. Balents, and P. L. McEuen, Nature 397, 598 (1999).

[11] B. A. Danilchenko, L. I. Shpinar, N. A. Tripachko, E. A. Voitsihovska, S. E. Zelensky, and B. Sundqvist, Appl. Phys. Lett. 97, 072106 (2010).

[12] B. Gao, A. Komnik, R. Egger, D. C. Glattli, and A. Bachtold, Phys. Rev. Lett. 92, 216804 (2004).

[13] M. Lazzeri, S. Piscanec, F. Mauri, A. C. Ferrari, and J. Robertson, Phys. Rev. Lett. 95, 236802 (2005).

[14] A. Javey, J. Guo, M. Paulsson, Q. Wang, D. Mann, M. Lundstrom, and H. Dai, Phys. Rev. Lett. 92, 106804 (2004).

[15] E. Pop, D. Mann, J. Cao, Q. Wang, K. Goodson, and H. J. Dai, Phys. Rev. Lett. 95, 155505 (2005).

[16] N. Bendiab, R. Almairac, J. L. Sauvajol, S. Rols, and E. Elkaim, J. Appl. Phys. 93, 1769 (2003).

[17] H. Kuzmany, W. Plank, M. Hulman, Ch. Kramberger, A. Gru“neis, Th. Pichler, H.

Peterlik, H. Kataura, and Y. Achiba, Eur. Phys. J. B 22, 307 (2001).

[18] A. Kukovecz, Ch. Kramberger, V. Georgakilas, M. Prato, and H. Kuzmany, Eur. Phys. J. B 28, 223 (2002).

[19] A. M. Rao, J. Chen, E. Richter, P. C. Eklund, R. C. Haddon, U. D. Venkateswaran, Y. K. Kwon, and D. Tomanek, Phys. Rev. Lett. 86, 3895 (2001).

[20] Z. Yu and L. E. Brus, J. Phys. Chem. B 105, 1123 (2001).

[21] S. Bandow, S. Asaka, S. Saito, A. M. Rao, L. Grigorian, J. Richter, and E. Eklund, Phys. Rev. Lett. 80, 3779 (1998).

[22] H. Rauf, T. Pichler, M. Knupfer, J. Fink, and H. Kataura, Phys. Rev. Lett. 93, 096805 (2004).

[23] C. Mora, R. Egger, and A. Alland, Semicond. Sci. Technol. 21, S46 (2006).

[24] S. Biermann, A. Georges, A. Lichtenstein, and T. Giamarchi, Phys. Rev. Lett. 87, 276405 (2001).

[25] A. A. Maarouf, C. L. Kane, and E. J. Mele, Phys. Rev. B 61, 11156 (2000).

[26] L. Balents, cond-mat/9906032V (1999).

[27] L. Venkataraman, Y. S. Hong, and P. Kim, Phys. Rev. Lett. 96, 076601 (2006).

[28] M. Lazzeri and F. Mauri, Phys. Rev. B 73, 165419 (2006).

[29] M. A. Kuroda, and J.-P. Leburton, Phys. Rev. B 80, 165417 (2009). 

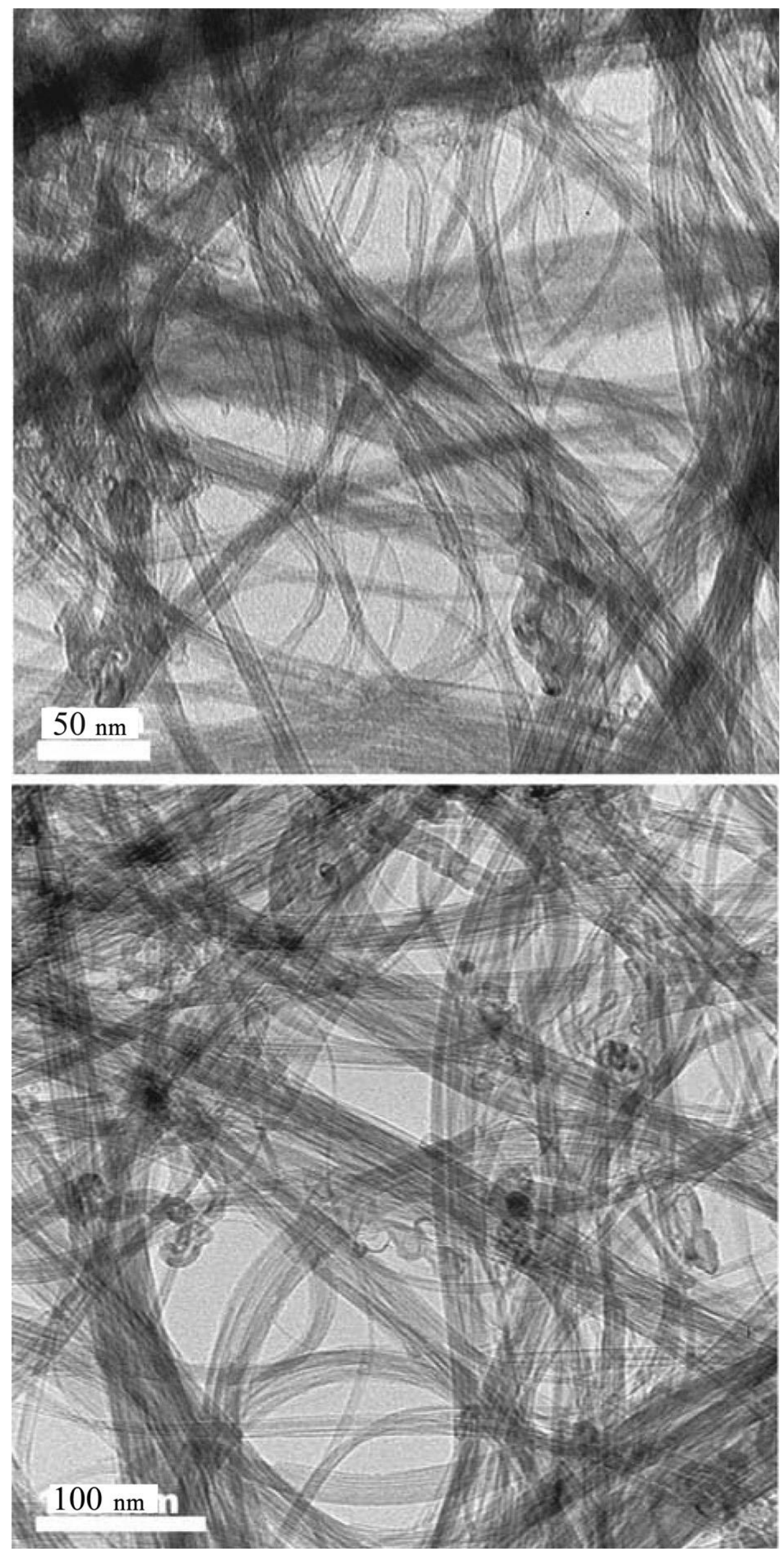

FIGURE 1 


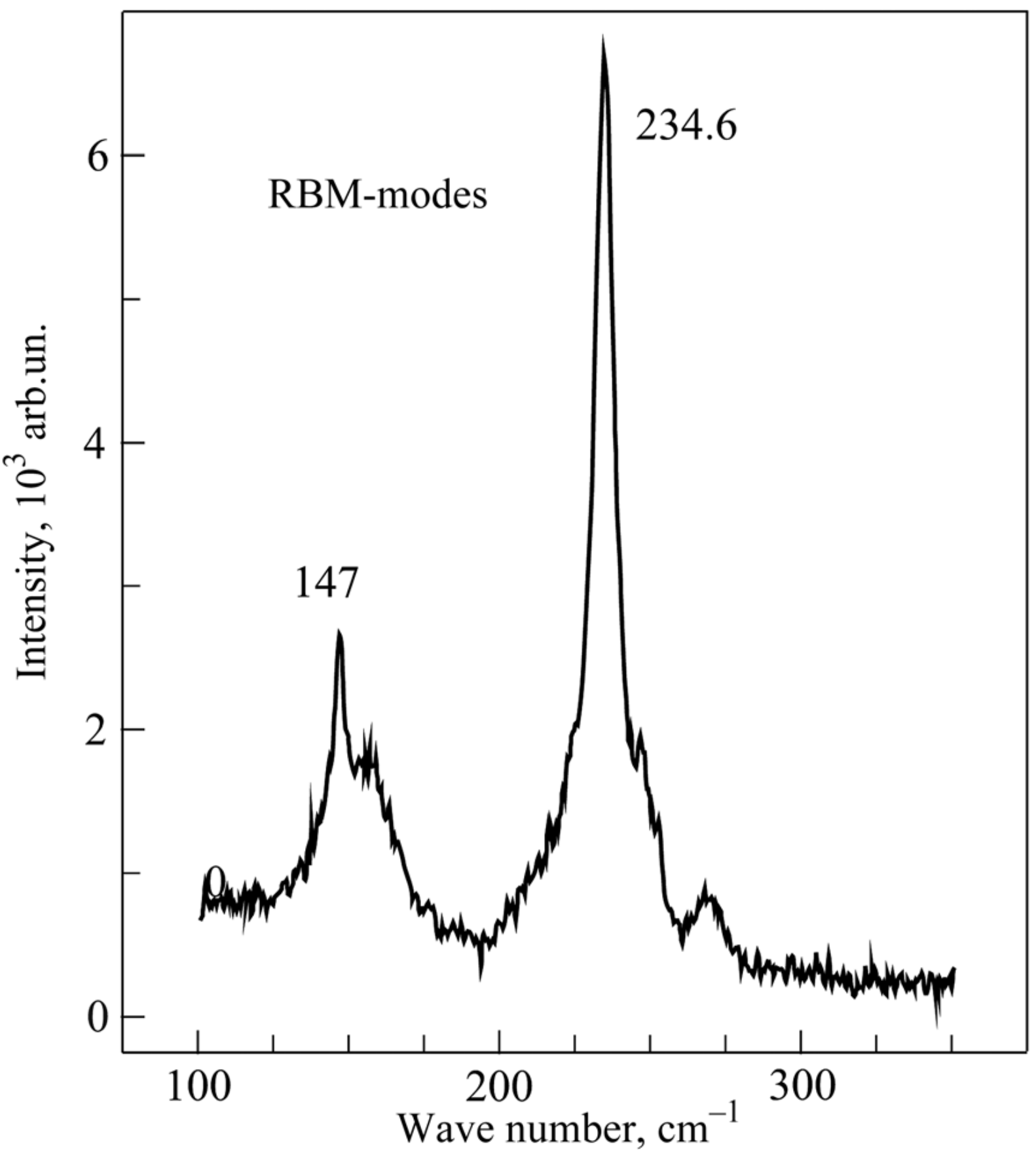

FIGURE 2 


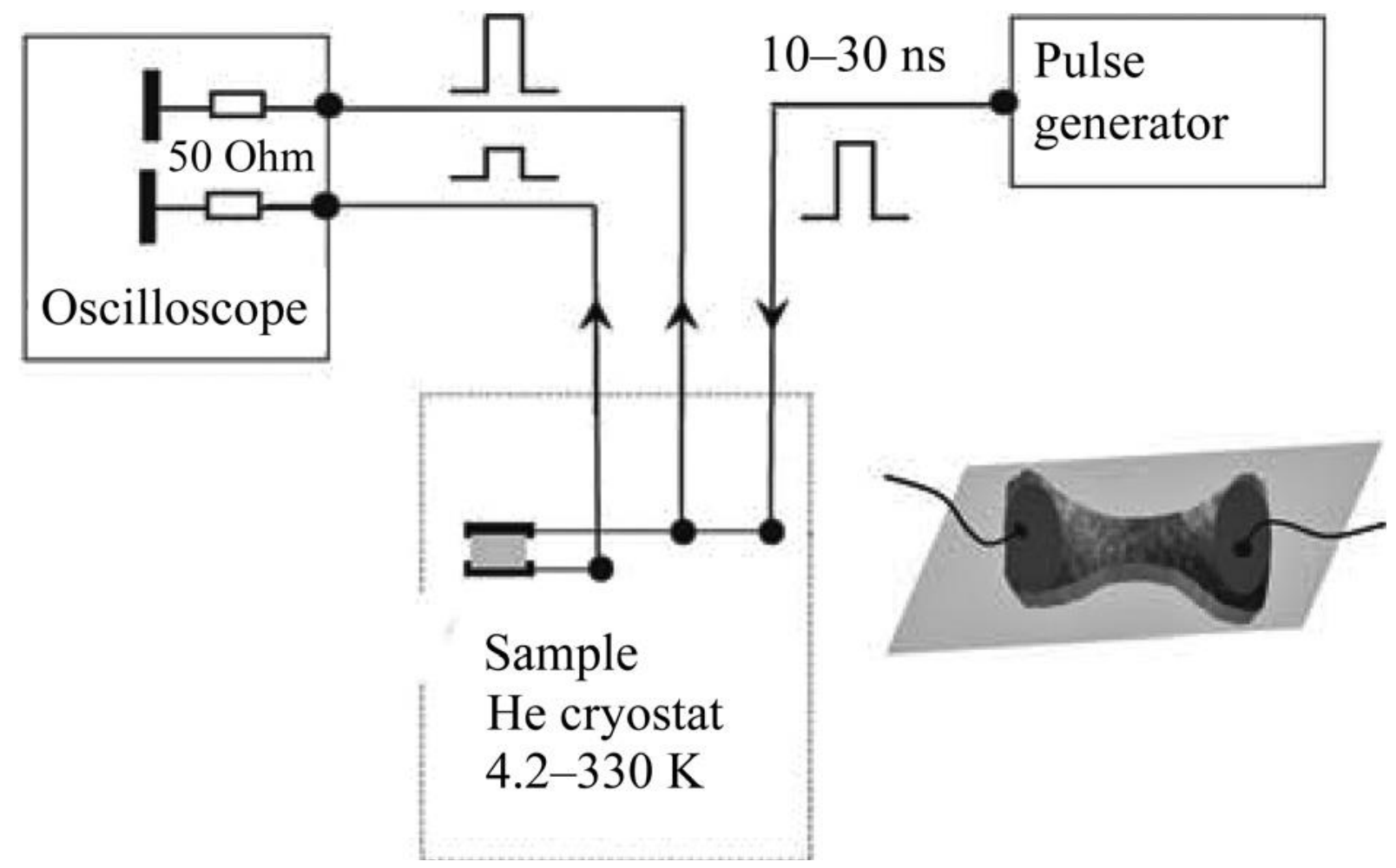

FIGURE 3 


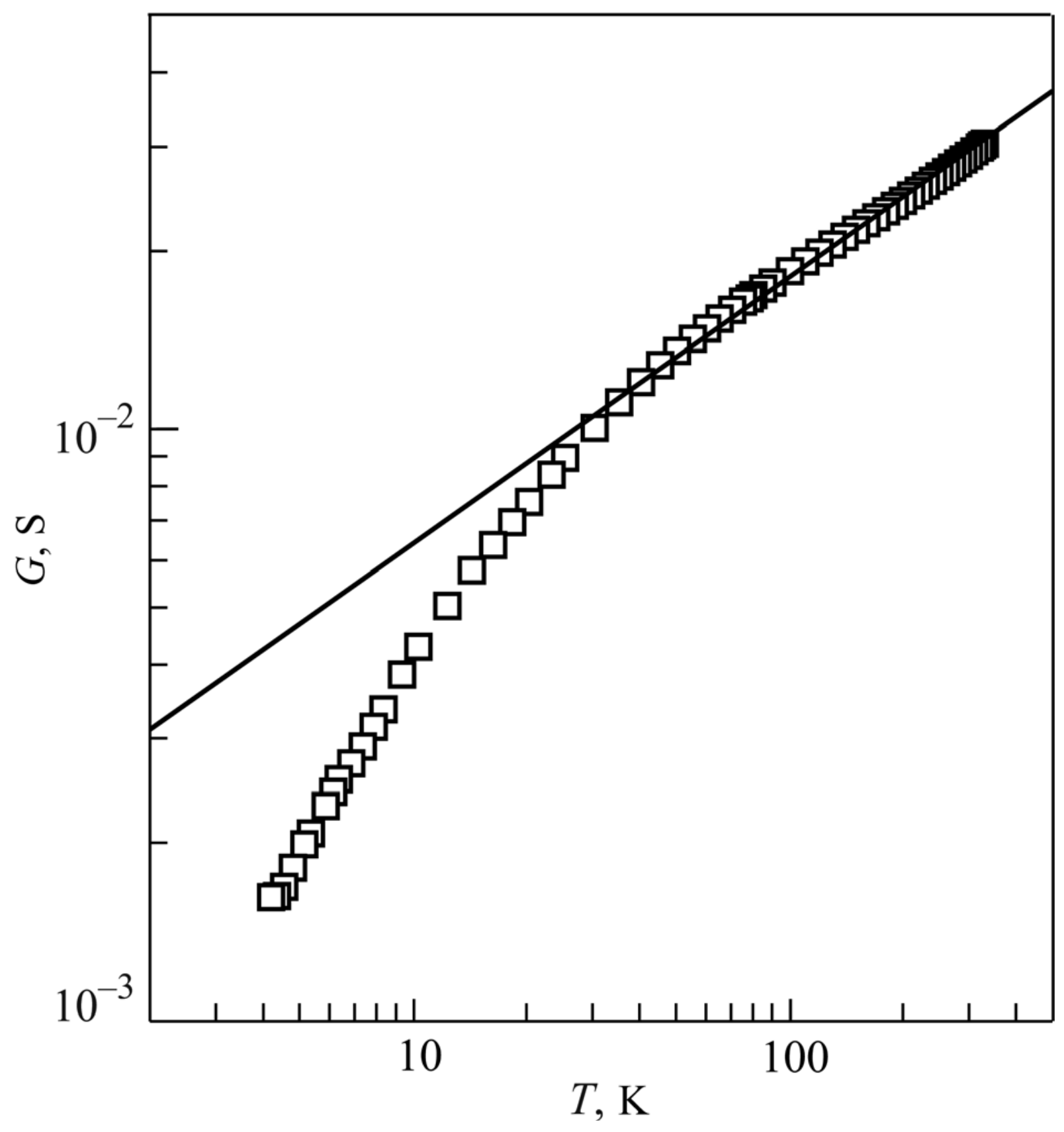

FIGURE 4 


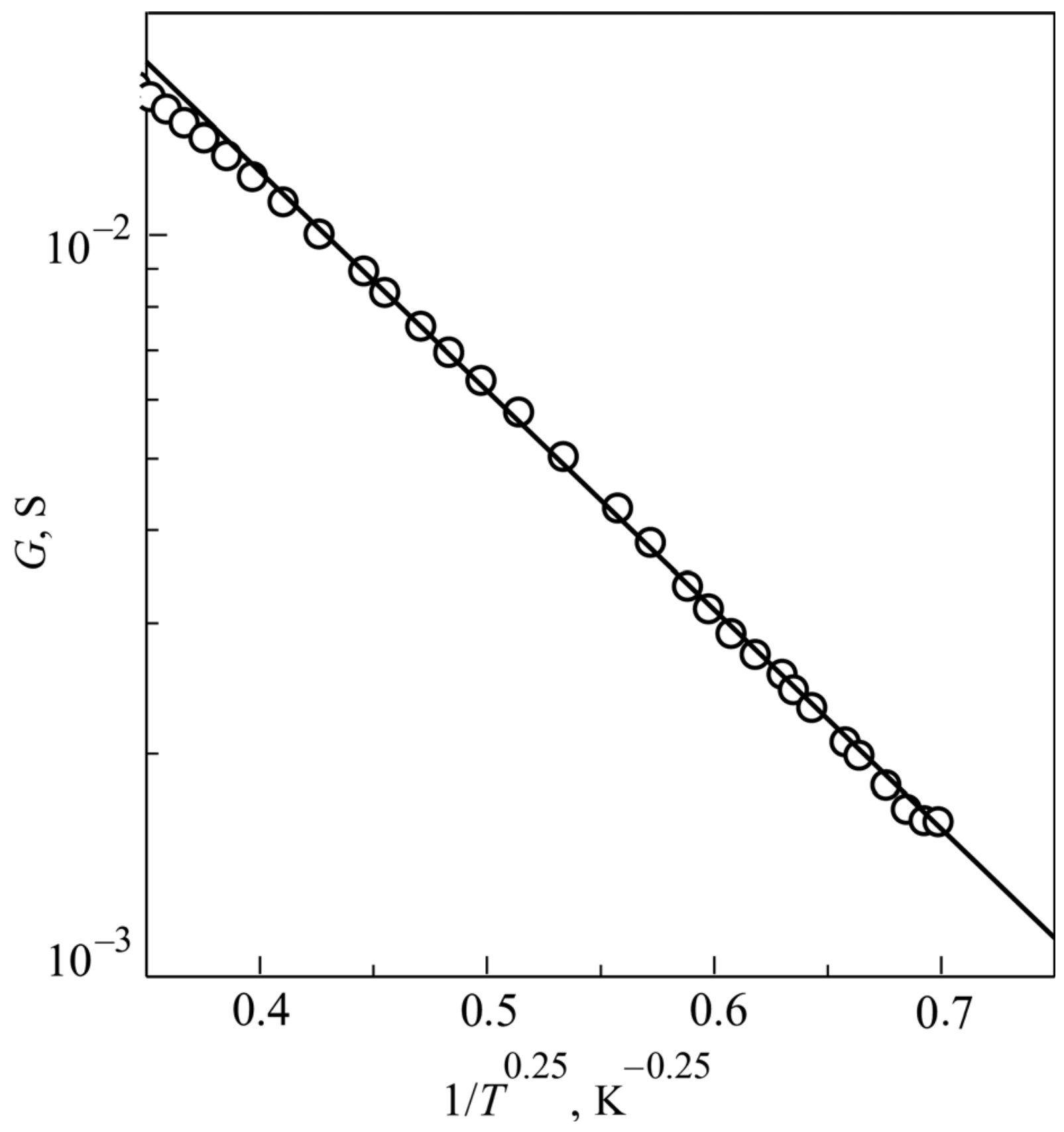

FIGURE 5 


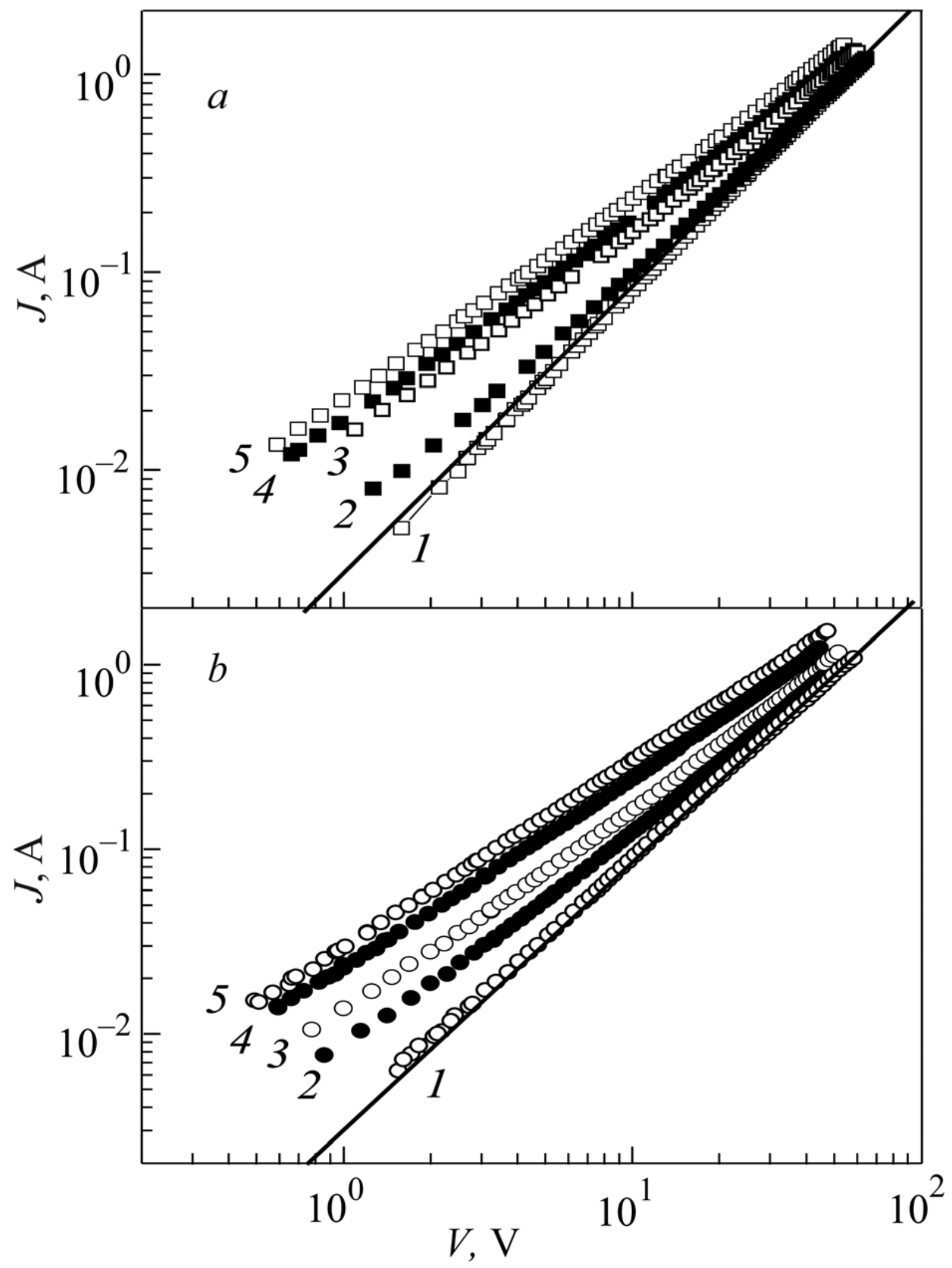

FIGURE 6 


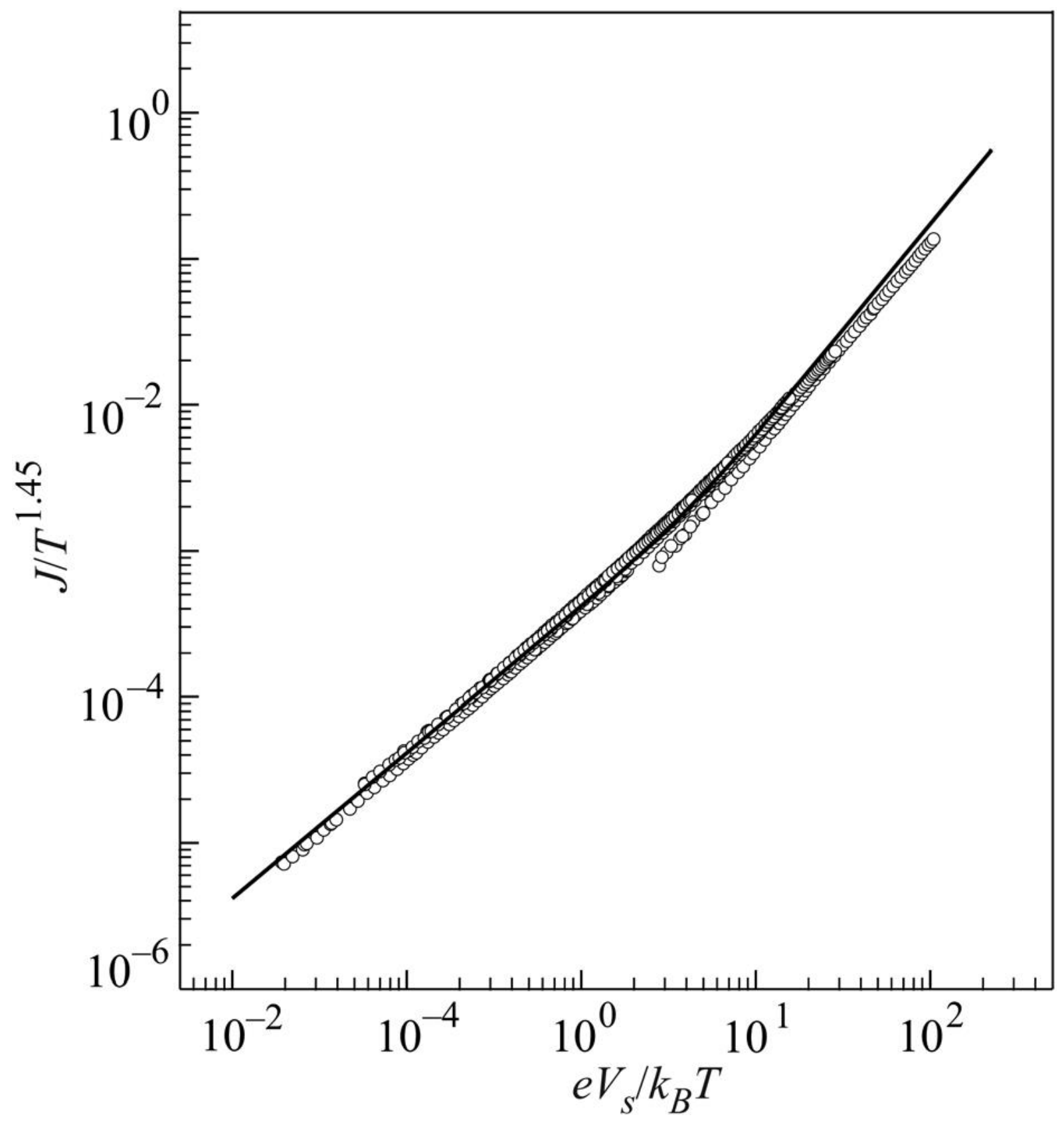

FIGURE 7 


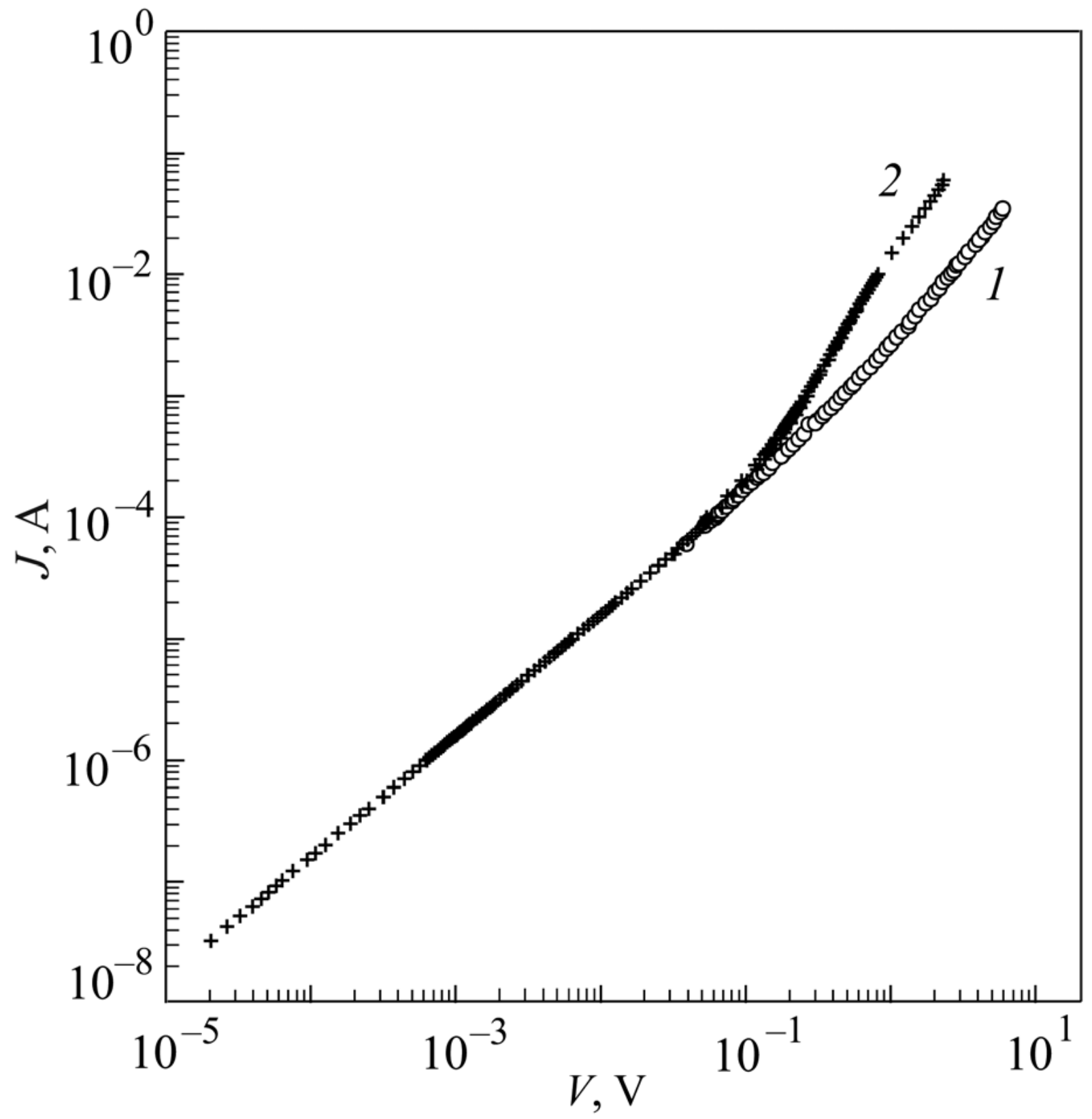

FIGURE 8 


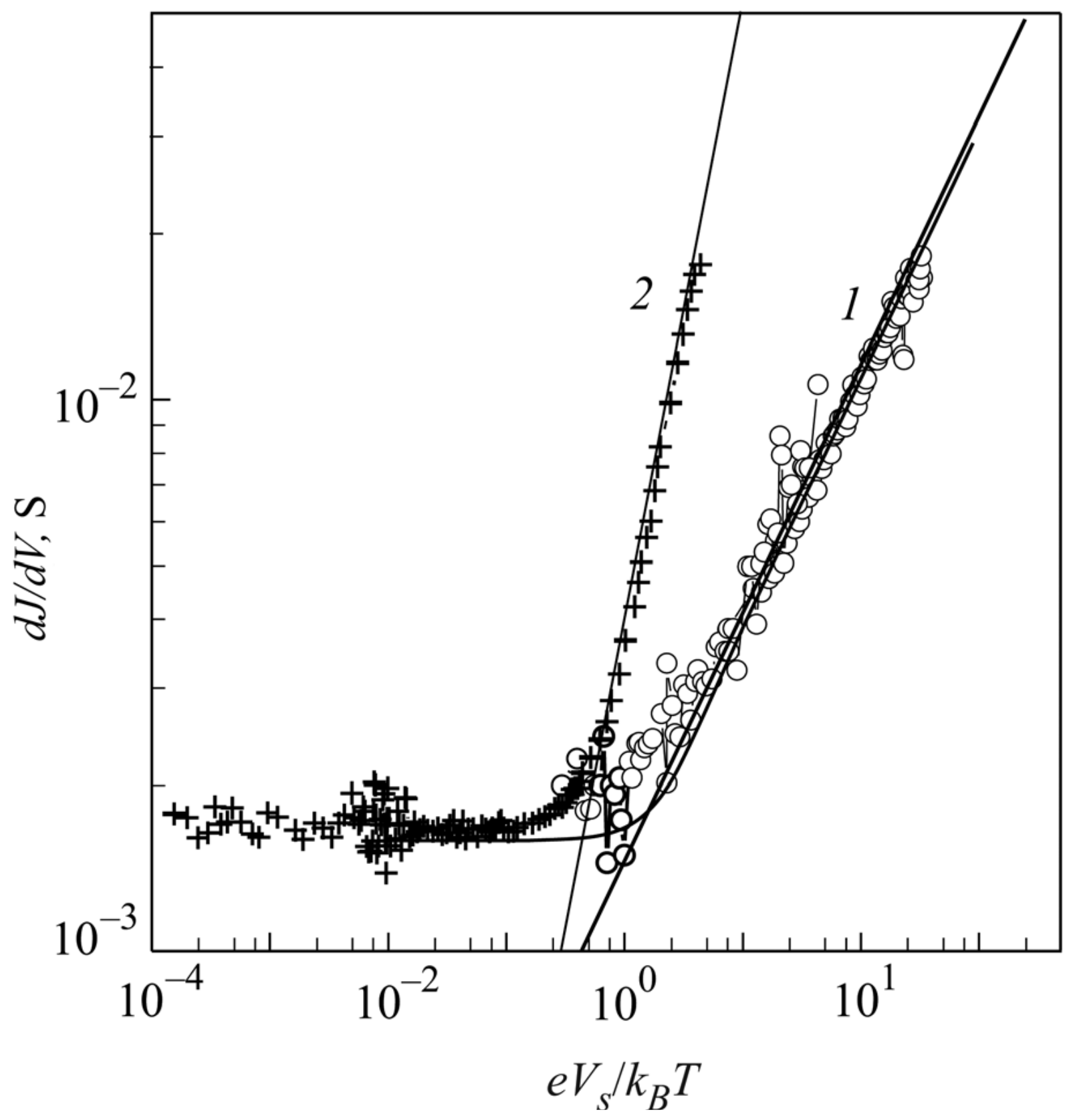

FIGURE 9 


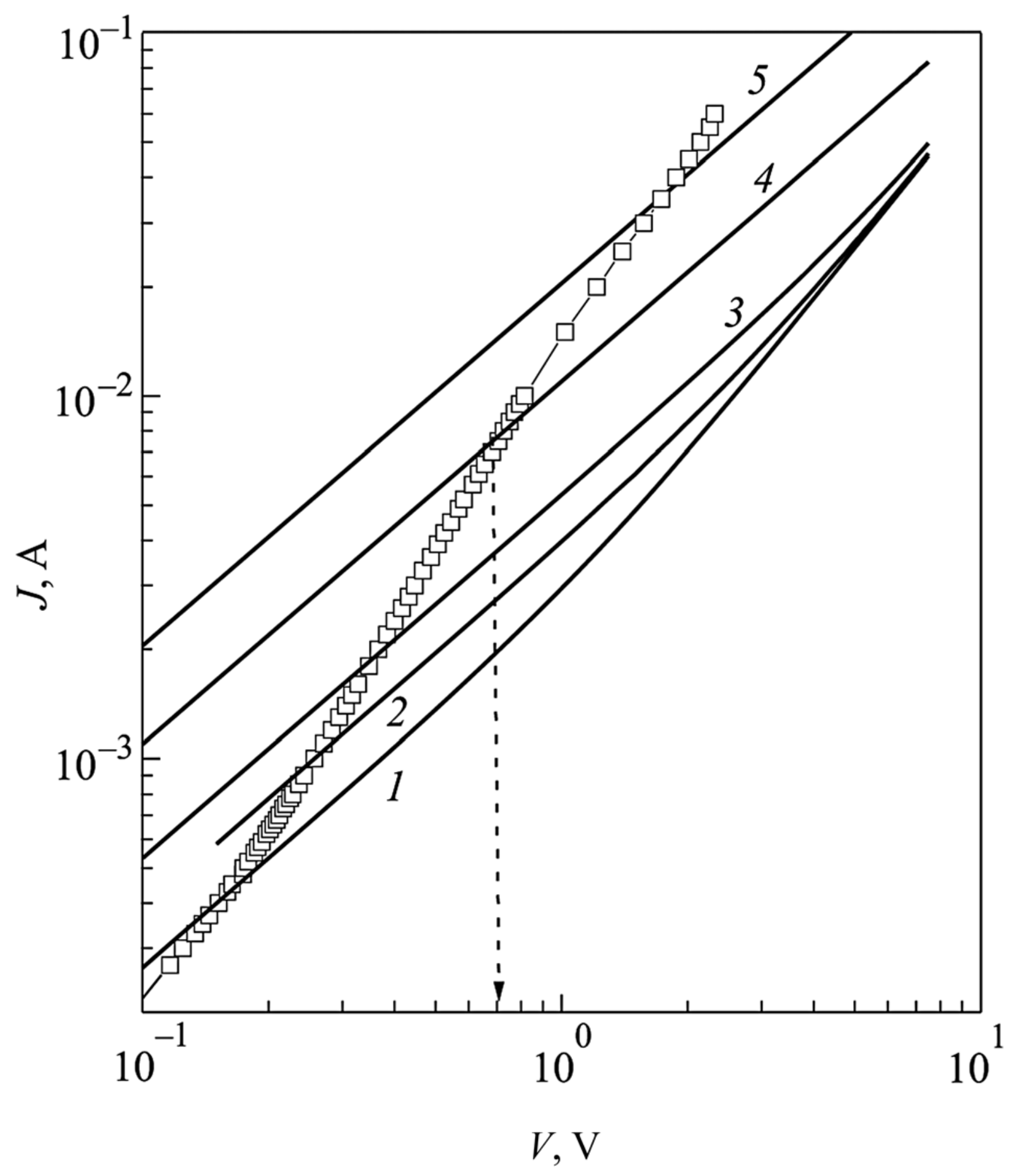

FIGURE 10 

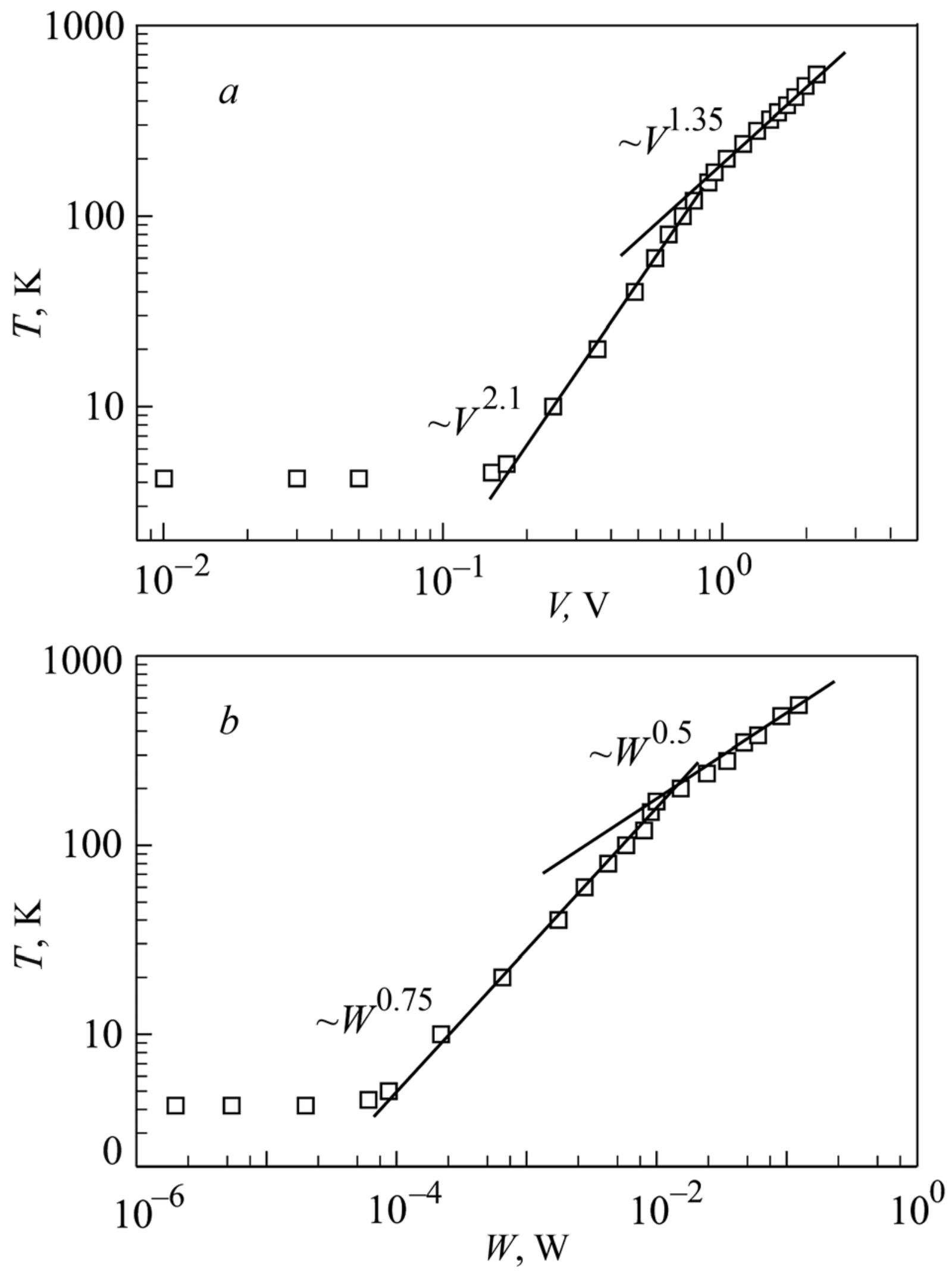

FIGURE 11 